\title{
Phenomenological study of the dynamics of pressure distribution in a gas flow in a long- distance pipeline
}

\author{
Mikhail Sukharev ${ }^{1, *}$, Olga Kochueva ${ }^{1, *}$ \\ ${ }^{1}$ National University of Oil and Gas «Gubkin University», Russia
}

\begin{abstract}
The paper is devoted to the modeling of unsteady gas flows in long pipelines. A widely used flow model in the form of a system of partial differential equations obtained by the methods of continuum mechanics is considered. The simplifying assumptions under which it is derived are discussed. It is stated that there is no experimental confirmation of the admissibility of these simplifications. Wave processes observed during the operation of the object are examined. The velocity of pressure waves propagation and attenuation was estimated, some physical phenomena, the causes of which are not clear from the available information, were identified. Actual observations are reconciled with the results of model calculations, some discrepancies between them are detected.
\end{abstract}

\section{Model of unsteady-state isothermal gas flow}

To describe flows in long-distance pipelines, it is customary to use one-dimensional models - systems of equations relating gas flow parameters (pressure $p$, flow $q$ and temperature $T$ ), as functions of two variables: space $x$ and time $t$. This paper examines quasi-isothermal model, where the unknown functions $p(x, t), q(x, t)$, are the mean values of pressure and flow rate with respect to normal cross-section of pipeline. The temperature is calculated after pressure and flow have been found.

The model includes a continuity equation (mass conservation equation) and a momentum equation

$$
\frac{\partial \rho}{\partial t}+\frac{1}{f} \frac{\partial M}{\partial x}=0, \frac{1}{f} \frac{\partial M}{\partial t}+\frac{\partial}{\partial x}\left(p+\rho w^{2}\right)=-\rho g \frac{d h}{d x}-\frac{\lambda}{\rho f^{2}} \frac{M|M|}{2 D}
$$

Here $M$ - the mass-flow rate of gas, $\rho$ - the gas density, $f$ - the cross-sectional area, $D$ - the inner pipe diameter, $w$ - gas flow rate, $h=h(x)$ - height of pipeline axis above sea level, $\lambda$ - hydraulic resistance coefficient, $g$ - gravity acceleration. The equation system (1) must be complemented with an equation of state

$$
\rho=p / z R T,
$$

\footnotetext{
*e-mail: mgsukharev@mail.ru ,olgakoch@mail.ru
} 
where $R$ - the gas constant, $z=z(p, T)$ - the compressibility factor. The arguments of the function $z$ are the average over the pipeline values of pressure and temperature. In practice instead of gas mass flow rate, standard flow rate $q=M / \rho_{s t}, \rho_{s t}=p_{s t} / R T_{s t}$ is used, that is flow rate is reduced to standard conditions $p_{s t}=1.01325 \cdot 10^{5} \mathrm{~Pa}, T_{s t}=293.15 \mathrm{~K}$. In this connection the equation system (1) will be written as

$$
\frac{\partial}{\partial t} \frac{p}{z R T}+\frac{\rho_{s t}}{f} \frac{\partial q}{\partial x}=0, \frac{\rho_{s t}}{f} \frac{\partial q}{\partial t}+\frac{\partial\left(p+\rho w^{2}\right)}{\partial x}=-\frac{p g}{z R T} \frac{d h}{d x}-\frac{\lambda \rho_{s t}^{2} z R T}{p f^{2}} .
$$

Some questions arise about the validity of assumptions used in equations derivation and their further development. The system (3) is sometimes simplified by omitting the component $\rho w^{2}$, since $\rho w^{2} \ll p$. The last relation is beyond doubt, but would be $\frac{\partial\left(\rho w^{2}\right)}{\partial x} \ll \frac{\partial p}{\partial x}$ (question №1)? The last component on the right side of the equation (3) is determined by frictional resistance. Its form was defined empirically from steady flow observations and was transferred to unsteady flow without experimental validation (the hypothesis of quasisteadiness). The question № 2: How far does this hypothesis take away from the truth? The quasi-isothermal hypothesis is used when transforming the system of equations (1) into the system of equations (3). The question № 3: When it is admissible? The question № 4: What approximations of function $z=z(p, T)$ should be used so that approximation error influence can be neglected? The question № 5: It is well known that such parameters as hydraulic resistance coefficient $\lambda$, heat transfer coefficient from pipeline to soil vary along the route. Considering them constant values we potentially allow some inaccuracy. What is the impact of this error on the result?

The strong answer to the questions posed could be obtained involving data from thoroughly conducted experiment preferably not in laboratory environment but on real-life object. These data generally could not be obtained when the basis of pipeline hydrodynamic theory have been formed. Furthermore, the scale of production facilities, pipes sizes, the operating pressure, the horsepower of gas-compressor units has grown tremendously. The models derived over half a century ago need to be verified for modern conditions and technologies of pipeline transport.

\section{Review of publications on topic}

The history of modeling nonsteady pipeline regimes lasts a few decades and it is extremely difficult to give any detailed overview of the papers on this topic. At first, only steady-state flows were studied [1], then papers appeared where nonsteady state regimes were also considered [2-26], research in the field of nonsteady state gas flows in long pipelines is still under way. In the monograph by Králik et al [6], a set of problems associated with the operation of gas supply systems, including transient regimes, is considered. The examples given in it were later used by some authors as a model for comparison. In papers [7-9], various aspects of the optimal operation of gas transport system (GTS) are considered. The study of nonstationarity of flow in large gas supply systems is of particular practical interest, as it allows us to characterize numerically the effect of a change in the amount of gas accumulated in pipes. This, in particular, is emphasized in the review by Ríos-Mercado R.Z., Borraz-Sánchez C. [10], which also contains a list of publications on the problem until 2014.

Recent years have been marked by a surge of interest in transient flows in gas networks due to the widespread (abroad) integrated (electric power plus gas) systems and systems with 
renewable energy sources, where gas serves as a reserve fuel. The main goal of the research is the coordinated control of large-scale power and gas supply systems in the daily operation cycle. Here we highlight the works of Zlotnik A., Chertkov M., Backhaus S. et al [11-13], Zhang N. et al [14], Yang J. et al [15], Behrooz H.A., Boozarjomehry R.B. [16-17]. In these papers, to imitate unsteady-state flows, the system of the hyperbolic type is most often used. That system is obtained from (3), where the term $\partial\left(\rho w^{2}\right) / \partial x$ is rejected as negligible. In [1618], the system of partial differential equations is approximated by a model with lumped parameters. In [17], the effect of information uncertainty on the results of optimization procedures is investigated, the degree of closeness of the calculated and actual regimes on the upcoming daily functioning cycle is estimated.

In [18], partial differential system is reduced ultimately to an algebraic system resembling a mathematical model of an electric power network. A very efficient model with lumped parameters was proposed in [19-20], where the parabolic system was taken as the basis for the approximation. In the Russian-language literature, the current state of the problem of modeling the transient gas flows is reflected in monographs [21-24].

\section{Initial data for analysis}

This paper analyses operational data from manometer measuring on twin gas pipeline under normal operation. The length of route is $176.9 \mathrm{~km}, 9$ measurement points equipped with manometers and thermometers are located close to each jumper between pipelines. The flow rate is measured at the end of the pipeline at the input of the compressor station. Two data sets transmitted via telemetry systems from measuring points to the control center of GTS were analyzed. The discrete nature of information leads to certain difficulties in its processing. An analysis of graphs revealed the presence of systematic errors in pressure gauges, which required pre-processing of measurements [25-26]. Analysis of the data allowed us to identify and investigate to an extent some physical phenomena (raising and lowering waves, "high-frequency" pressure fluctuations) that occur during the flow of gas in the pipelines.
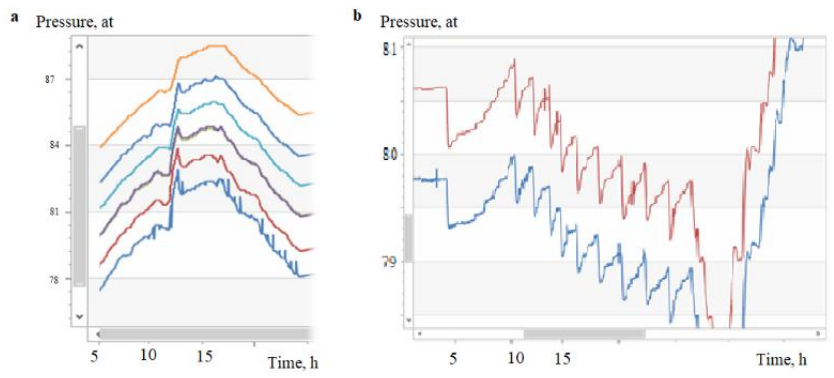

Fig. 1. a - a pressure peak propagating in the direction against the gas flow. b - "highfrequency" pressure fluctuations

a) Raising and lowering waves (peaks and troughs). Figure 1a illustrates a wave when within a relatively short period pressure increased by about 2.7 at, then quickly dropped by almost 1 at. Figure demonstrates that peak moved against the flow, meanwhile both the "height" of peak and following drop were damped. Peaks and troughs can be classified as solitary waves. The curves in Figure 1 depict measured pressure values at adjacent metering points.

b) "High-frequency" oscillations. Another phenomenon is oscillatory process, an example is given in the figure $1 \mathbf{b}$. 


\section{Classification of causes of nonstedy-state gas flow in pipes}

Pressure peaks and "high-frequency" oscillations outlined above relate undoubtedly to nonsteady processes. The reasons of unsteadiness are following: 1) changes in ambient temperature; 2) hard deposits (sand, slimes) inside the pipe, condensate dropout, hydrate formation; 3) volatility of consumer demand; 4) switching on and off the equipment; 5) the formation of relatively small through holes (cracks, eroded holes) in the pipe body; 6) small disturbances (acoustic vibrations); 7) guillotine pipe break.

The reasons 1, 2, and 3 cause slow evolution of flow parameters. To examine them in some cases it suffices to use steady and quasi-steady models, in other cases dynamics of accumulated in pipes gas should be considered. To cover this requirement lumped parameter model $[19,20,22]$ is developed, in calculating process it is significantly more effective than partial differential equation model (equations (3)). The disturbances of the 4th group have not been phenomenologically investigated so far, anyhow the authors are not aware of relevant publications. The present study focuses on the analysis of pressure surges, recessions and "high-frequency" oscillations related exactly to this group.

The causes of the 5th and 7th groups in the study period did not appear. The propagation of small-scale disturbances (6th group) can be analyzed involving methods of continuum mechanics [23]. They are described by system (3), which is of a hyperbolic type.

\section{Speeds of "middle waves" and "high-frequency" oscillations}

We made calculations to estimate the velocity of pressure waves propagation. The scatter of the results - from $151 \mathrm{~m} / \mathrm{s}$ to $220 \mathrm{~m} / \mathrm{s}$ - is large. It could be explained, first of all, due to the discreteness of pressure fixation. The estimations are substantially different from physical speed of gas particle movement and from sound velocity $c_{s}$. Velocity of gas particle movement equals $10-20 \mathrm{~m} / \mathrm{s}$ at the level of parameters, which are common to the operating conditions for onshore gas pipelines. Various estimations of the sound velocity $c_{s}$ in gas flow range between $320-380 \mathrm{~m} / \mathrm{s}$ [23].

We note a certain similarity between solitary waves (peaks and troughs) with solitons waves in open channels that have attracted the attention of researchers for nearly 200 years. The outlined phenomena differ from the soliton by a noticeable decrease in the amplitude of oscillations with time.

"High-frequency" pressure oscillation can also propagate towards and against the gas flow. Estimates of oscillation propagation velocity are about $200 \mathrm{~m} / \mathrm{s}$ and have been less volatile.

\section{Comparison of actual measures with the results of numerical modeling}

To verify the models, which are currently used to simulate nonsteady flow in gas pipelines, calculations were carried out using software package "Vesta». In «Vesta» the model of transient flow is system (3), where however the derivative $\partial\left(\rho w^{2}\right) / \partial x$, is omitted. The initial and boundary conditions for calculations were chosen in such a way as to simulate a pressure wave. The operational data are compared with the calculations in the figure 2 . The curves at the left side show the actual pressure change. The wave moves against gas flow direction. In 
accordance with this, the curves are located: of the two adjacent curves, the lower one corresponds to the measuring point, further downstream from the beginning of the section.

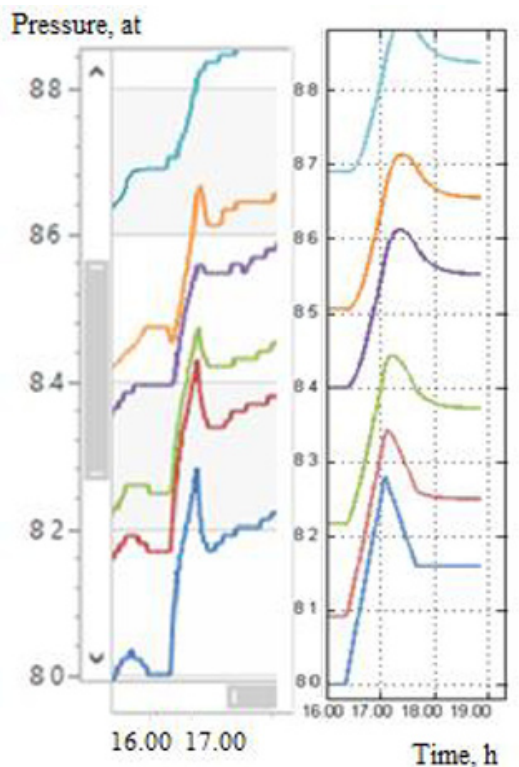

Fig. 2. The dynamics of pressure peak propagation. At the left - actual manometric measuring, at the right the results of computer simulation At the beginning of the pipeline (with coordinate $x=0$ ) either pressure or flow rate is assigned a constant value. At the end of the pipeline (with coordinate $x=1$ ) pressure as function of $t$ was set. This function simulated the peak: initially linear growth of the pressure then linear drop to the certain level and stabilization at that level. The bottom curve in the figure 2 on the right shows the given function $p(1, t)$.

Let us compare the observed (figure 2, at the left) and calculated (figure 2, at the right) flow regimes. They have some similarities. A closer look reveals the substantial difference between that curves. The actual blurring of the peak is much faster than on the model. As can be seen from fig. 2, on the left, when approaching the $1^{\text {st }}$ metering point, the peak practically disappeared: the pressure rose and stabilized. According to the computational model (figure 2, at the right) although the height of the peak is decreasing, but undoubtedly is still noticeable, the pressure has not stabilized. Furthermore, in fact the peak has the shape of an acute angle up to the second current-wise measuring point. In the computational model flattening out of the peak takes place, much more substantive as the wave moves. These model deviations from fact testify to the incomplete adequacy of the model and to the advisability of its improving.

\section{Conclusions}

The graphs of pressure changes over several days recorded by the whole complex of metering points located on a twin gas pipelines are analyzed. This information is used for the critical analysis of mathematical models of transient gas flow in long pipelines. Based on the results of the analysis, the following approaches are proposed to correct (refine) the one-dimensional flow model in order to more adequately describe real processes: the preservation of the often rejected term in the equation of momentum, the rejection of the hypothesis of quasistationarity.

It has been established that in the operating regimes of loaded gas pipelines the following phenomena not previously described in the literature take place: sharp bursts and drops in pressure, propagating as solitary waves with damped amplitude, and periodic oscillatory processes. Certain similarities of solitary waves with solitons - waves in open channels have been noted. The velocity of solitary waves and periodic processes was estimated. The estimation of the velocity of solitary waves and for batch processes was conducted. The observed wave propagation velocities are an order of magnitude greater than the velocity of movement of gas particles and 2-3 times less than the speed of sound.

Information systems of trunk pipelines provide huge amounts of real-time information, which, however, is not fully used, though it could be applied to improve the procedures for control of gas transportation systems. Particularly it allows to increase the degree of validity 
of unsteady gas flow models. Refining gas flow models in main pipelines, development of new information technologies for pre- processing and analysis of incoming information will help to improve the quality of dispatch solutions, will open up new opportunities for forecasting the occurrence of abnormal situations including automatic detection of leaks and will contribute to the improvement of operational control of the gas transmission system as a whole.

\section{References}

1. T.R. Weymouth, Problems in natural gas engineering, 34 (Trans. ASME, 1912)

2. T.W. Johnson, W.B. Berwald, Flow of natural gas through high-pressure transmission lines. Bureau of mines, 6 (1935)

3. I.A. Charnyj, Unsteady motion of real fluid in pipes (Nedra, Moscow, 1975) [In Russian]

4. M.G. Sukharev, Ye.R. Stavrovskiy, Optimization of gas transportation systems (Nedra, Moscow, 1975) [In Russian]

5. O.F. Vasil'yev, E.A. Bondarev, A.F. Voyevodin, M.A. Kanibolotskiy, Non-isothermal gas flow in pipes (Nauka, Novosibirsk, 1978) [In Russian]

6. J. Králik, P. Stiegler, Z. Vostrý, J. Zaworka, Dynamic Modeling of Large-Scale Network with Application to Gas Distribution (Elsevier, Amsterdam-Oxford-NY-Tokyo, 1988)

7. A.J. Osiadacz, Proc. 26th annual meeting of Pipeline Simulation Interest Group (1994)

8. K. Ehrhardt, M. C. Steinbach, ZIB Report ZR-03-46 (Berlin, 2003)

9. I. Cameron, Proc. 31st Annual Meeting PSIG (Pipeline simulation Interest Group, 1999)

10. R.Z. Ríos-Mercado, C. Borraz-Sánchez, Applied Energy, 147, 536 (2014)

11. A. Zlotnik, S. Dyachenko, S. Backhaus, M. Chertkov, Proc. Dynamic Systems and Control Conference, 3, 1 (2015)

12. A. Zlotnik, M. Chertkov, S. Backhaus, arXiv:1504.02505 2015 (2015)

13. A. Zlotnik, L. Roald, S. Backhaus, M. Chertkov, G. Ande, Proc. American Control Conference, 7478 (2016)

14. N.Y. Chiang, V.M. Zavala, Applied Energy, 168, 226 (2016)

15. J. Yang, N. Zhang, Ch. Kang, P. Pinson, Proc. Conf.: IEEE PES General Meeting (2017)

16. H.A. Behrooz, R.B. Boozarjomehry, Journal of Natural Gas Science and Engineering, 22, $551(2015)$

17. H.A. Behrooz, R.B. Boozarjomehry, Energy (2017) [Accepted manuscript]

18. G.R. Price, R.K. McBrien, S.N. Rizopoulos, H. Golshan, Journal of Offshore Mechanics and Arctic Engineering, 121, 131 (1999)

19. M.G. Suharev, R.V. Popov, Izvestiya RAN. EHnergetika, 2, 150 (2015) [In Russian]

20. M.G. Sukharev, K.O. Kosova, R.V. Popov, Energy (2018)

21. S.A. Sardanashvili, Calculation methods and algorithms (pipeline gas transportation) (Neft' i gaz, Moscow, 2005) [In Russian]

22. M.G. Suharev, R.V. Samojlov, Analysis and control of steady and unsteady modes of gas transportation (Izd. Centr RGU nefti i gaza, Moscow, 2016) [In Russian]

23. M.V. Lur'e, Theoretical foundations of pipeline transport of oil, petroleum products and gas (Nedra, Moscow, 2017) [In Russian]

24. E.A. Bondarev, A.F. Voyevodin, Solving problems of pipe hydraulics in natural gas production and transportation systems (SO RAN, Novosibirsk, 2017) [In Russian]

25. M. Sukharev, Ks. Kosova, E3S Web Conf., 25, 02001 (2017)

26. M.G. Suharev, K.O. Kosova, Trudy RGU nefti i gaza, 2 (2017) [In Russian] 